\title{
ON THE GAUSS CURVATURE OF MINIMAL SURFACES $\left({ }^{1}\right)$
}

\author{
BY \\ ROBERT OSSERMAN
}

1. Summary of results. The following is known: let $S$ be a minimal surface defined by $z=f(x, y)$ over the region $D: x^{2}+y^{2}<R^{2}$, and let $p$ be the point of $S$ over the origin. Let $W=\left(1+f_{x}^{2}+f_{y}^{2}\right)^{1 / 2}$ at $p$. Then the Gauss curvature $K$ at $p$ satisfies $|K| \leqq c / R^{2} W^{2}$. The best numerical value of $c$ known previously was 12.25 . This inequality is simultaneously sharpened and generalized. First of all, it is proved that if $d$ is the distance along the surface from $p$ to the boundary of $S$, then $|K| \leqq 8 / d^{2} W^{2}$. Clearly $d \geqq R$. Furthermore this sharper bound holds much more generally where $D$ is any simply-connected region, $p$ any point of $S$, and $d$ and $W$ are as before. The constant 8 cannot be much improved, since for arbitrary $d>0$ there exists a surface with $W=1$ such that $|K|=64 / 9 d^{2}$. It is further shown that among surfaces with $W=1$ the slightly stronger inequality $|K| \leqq 64 / 9 d^{2}$ holds, and by the above example this is best possible. All of these results are extended to cases where $S$ is not representable in the form $z=f(x, y)$. The methods used also yield a number of other results for the class of surfaces considered.

2. Introduction. The majority of results in this paper are derived from the following observation. Given a point $p$ on a minimal surface, one may assign to each point of a suitable neighborhood $N$ of $p$ two complex variables $\zeta$ and $w$, such that the correspondence between $\zeta$ and $w$ is analytic, and for the Gauss curvature $K$ at each point of $N$ we have the formula

$$
K=-\left[\frac{4}{\left(1+|w|^{2}\right)^{2}}\left|\frac{d w}{d \zeta}\right|\right]^{2} \text {. }
$$

This allows us to translate elementary properties of analytic functions into geometric statements about minimal surfaces.

The variable $w$ is obtained simply by making the Gauss spherical map of $N$ into the unit sphere and following this by a stereographic projection into the complex w-plane. This technique has been used since the early days in the theory of minimal surfaces, and it was applied by E. Hopf [4] to the kind of problem which we shall consider.

The variable $\zeta$ was introduced by the author [9] and stems from the mapping constructed by Nitsche [5] in the particular case of a minimal surface which can be represented in the form $z=f(x, y)$. There are two main differences between our approach and the one used by Nitsche. First, we study

Presented to the Society, September 2, 1959; received by the editors July 21, 1959.

(1) This work was supported by the Office of Ordnance Research, U. S. Army. 
directly the behavior of the map of the surface into the $\zeta$-plane, whereas Nitsche's method uses properties of the map of the $x, y$-plane into the $\zeta$-plane. Next, by using this direct approach, we are able to define $\zeta$ by a method which does not require the neighborhood $N$ to have representation of the form $z=f(x, y)$. Using the former we are able to sharpen Nitsche's results, and using the latter, to generalize them.

In addition to the results involving formula (1), a number of consequences can be drawn using the variable $\zeta$ alone. Some of these are given in [11]. In $\S 6$ of the present paper we indicate several results which follow from our general method, but which are not directly concerned with the main subject of this paper.

$\S \S 3,4$, and 5 deal with the following problem: given a point $p$ of a minimal surface and a neighborhood $N$ of $p$, to determine a bound for the Gauss curvature at $p$ in terms of geometric properties of the neighborhood $N$. Various results of this kind are known $[3 ; 4 ; 7 ; 10]$. Theorem 1 below is another such result, but in contrast to all the previous ones the bound obtained is precise. Namely, for all admissible values of the parameters which occur, we are able to exhibit surfaces for which the extremal value of $K$ is attained.

In Theorem 2 we treat the same problem, but with different restrictions on the neighborhood $N$. In this case we have not been able to determine the extremal surfaces, and the expression which we obtain for the bound on $K$ is a rather complicated one. However, what Theorem 2 lacks in elegance it makes up for in generality, since it includes as special cases all previous results of the kind indicated above. This is shown in $\$ 5$. We may summarize the situation as follows:

First of all, Theorem 2 contains both Theorem 1 of the present paper and also a sharper version of a theorem proved earlier [10]. Secondly, like the latter theorem, it extends the results of [3], [4], and [7] by weakening the restriction on the neighborhood $N$. Namely, instead of assuming that $N$ can be represented in the form $z=f(x, y)$ we require only that the normal directions to $N$ be bounded away from some fixed direction, and we replace the assumption that $N$ project onto a disk by the requirement that $N$ be simply-connected. Finally, if we apply the inequality of Theorem 2 to the case where $N$ does project onto a disk, we obtain an inequality which is stronger than the previous ones.

3. Preliminary results. Our method of proof is a refinement of one used in a previous paper [10]. As in that paper, the argument is based on the following fundamental lemma:

LEMMA 1. Let $p$ be a point on a mininal surface, and $N$ a simply-connected neighborhood of $p$. Assume that the Gauss spherical map of $N$ into the unit sphere $\Sigma$ omits at least one point of $\Sigma$, and project $\Sigma$ stereographically from that point onto the complex w-plane. This assigns to each point $q$ of $N$, a complex number $w(q)$. 
Then there exists a mapping of $N$ into the complex $\zeta$-plane which is one-to-one conformal in a neighborhood of each point $q$ of $N$, and which satisfies

$$
\left[\frac{|d \zeta|}{d s}\right]_{q}=\frac{2}{1+|w(q)|^{2}}
$$

throughout $N$, where ds is the element of arc length on the surface.

A proof of this lemma is contained in a previous paper [9]. We do not reproduce the proof here, but we make the following remarks.

1. The map of $N$ into the $\zeta$-plane will not in general be globally one-toone.

2. In the case that $N$ has a one-to-one parallel projection onto a plane region, i.e., if by a suitable choice of coordinates we may write $N$ in the form $z=f(x, y)$, then our map of $N$ into the $\zeta$-plane is exactly the one given by Nitsche [5].

3. Several of the preliminary results in [9] are stated in a general form and make use of rather powerful tools, such as the existence of isothermal parameters for a general Riemannian metric, and the Koebe uniformization theorem. However, neither of these is needed if we merely wish to prove the above lemma, since the existence of local isothermal parameters on a minimal surface is elementary, (one could, for example, use Nitsche's mapping,) and by the simple connectivity of $N$ and the fact that a map satisfying (2) is defined up to a Euclidean motion in the $\zeta$-plane, one can piece together local maps into the $\zeta$-plane to get a map of the whole neighborhood $N$.

4. The neighborhood $N$ is allowed to have self-intersections of an elementary character. Thus, the condition that $N$ be simply-connected does not refer strictly to $N$ considered as a point set in space, but rather as a surface which may contain certain arcs along which each point is counted twice, with distinct neighborhoods intersecting along the arc.

In addition to the above remarks, we recall two well-known properties of the Gauss spherical map in the case of minimal surfaces. First, the map of $N$ into $\Sigma$ is conformal, and second, the stretching factor is given in terms of the Gauss curvature by the formula

$$
\frac{d \sigma}{d s}=|K|^{1 / 2}
$$

where $d \sigma$ is the element of arc length on $\Sigma$.

We may always, without loss of generality, choose coordinates so that the point on $\Sigma$ from which we project corresponds to the direction of the positive $z$-axis. The equations of stereographic projection then take the form

$$
x=\frac{2 u}{|w|^{2}+1}, \quad y=\frac{2 v}{|w|^{2}+1}, \quad z=\frac{|w|^{2}-1}{|w|^{2}+1},
$$


where $w=u+i v$ corresponds to the point $(x, y, z)$ of $\Sigma$.

Furthermore, as is well known and easily deduced from (4), we have for the line element $d \sigma$ on $\Sigma$,

$$
d \sigma=\frac{2|d w|}{1+|w|^{2}} .
$$

Combining (2), (3), and (5), we obtain the basic relation (1).

\section{The precise bound.}

Lemma 2. Let $p$ be a point of a minimal surface and $N$ a simply-connected neighborhood of $p$ having the property that for some $\beta<\pi$, all normals to points of $N$ make an angle of less than $\beta$ with the normal at $p$. Let the distance along the surface from $p$ to the boundary of $N$ be at least $d$. Define the map of $N$ into the w-plane as in Lemma 1, using stereographic projection from that point of $\Sigma$ which corresponds to the direction opposite to the normal at $p$. Construct the map of $N$ into the $\zeta$-plane using Lemma 1 . Let the image of $p$ be $\zeta_{0}$. Then the inverse map is well defined in a disk $\left|\zeta-\zeta_{0}\right|<\rho$, where

$$
\rho \geqq 6 d /\left(3+r^{2}\right), \quad r=\tan \frac{\beta}{2} .
$$

Proof. We may assume by a translation of the $\zeta$-plane that $\zeta_{0}=0$. Since the map of $N$ into the $\zeta$-plane is locally one-to-one, the inverse map is well defined in a neighborhood of the origin. If this map can be extended to the whole $\zeta$-plane, then the lemma is certainly true. Otherwise there is a largest disk, $|\zeta|<\rho$, in which it is defined. There must then be a point $\zeta_{1}$ with $\left|\zeta_{1}\right|=\rho$ such that the inverse map cannot be extended to a neighborhood of $\zeta_{1}$. Let $L$ be the line segment joining 0 to $\zeta_{1}$ and let $C$ be the corresponding curve in $N$. Then the length of $C$ must be at least $d$, since otherwise $C$ would lead from the point $p$ to some interior point $q$ of $N$, and the one-to-one map in the neighborhood of $q$ would allow us to extend the inverse map to a neighborhood of $\zeta_{1}$.

We therefore have

$$
d \leqq \int_{C} d s=\int_{L} \frac{1+|w|^{2}}{2}|d \zeta|
$$

using the defining property (2) of the map of $N$ into the $\zeta$-plane. But the way we have constructed our map into the $w$-plane, the image of $p$ is the origin, and all of $N$ maps into a disk of radius $r=\tan (\beta / 2)$. We therefore have an analytic map of the disk $|\zeta|<\rho$ into $|w|<r$ with the origin mapping into the origin, and by Schwarz' Lemma, $|w| \leqq r|\zeta| / \rho$ at each point. Inserting this in (7), we obtain

$$
d \leqq \frac{1}{2} \int_{L}\left(1+r^{2}|\zeta|^{2} / \rho^{2}\right)|d \zeta|=\frac{1}{2} \int_{0}^{\rho}\left(1+r^{2} t^{2} / \rho^{2}\right) d t=\frac{1}{2}\left(1+r^{2} / 3\right) \rho,
$$

which is precisely (6). 
THEOREM 1. Let $p$ be a point on a minimal surface and $N$ a simply-connected $\left({ }^{2}\right)$ neighborhood of $p$ having the property that for some number $\beta<\pi$ the normal at $p$ makes an angle of less than $\beta$ with the normal at each point of $N$. Let the distance along the surface from $p$ to the boundary of $N$ be at least $d$. Then the Gauss curvature $K$ at $p$ satisfies

$$
|K| \leqq \frac{1}{d^{2}}\left[\frac{2}{3} r\left(3+r^{2}\right)\right]^{2}, \quad r=\tan \frac{\beta}{2} .
$$

Furthermore, inequality (8) is precise. For each, choice of $\beta$ and $d$, with $0<\beta<\pi, d>0$, there exists a minimal surface, a point $p$, and a neighborhood $N$ satisfying the hypotheses, for which equality holds in (8).

Proof. We have constructed in the proof of Lemma 2 an analytic map of the disk $|\zeta|<\rho$ into $|w|<r, r=\tan (\beta / 2)$, where $\rho$ satisfies (6) and the origin is mapped into the origin. By Schwarz' Lemma, $|d w / d \zeta|_{\zeta=0 \leqq r / \rho}$. Using (1) with $w=0$, we obtain

$$
|K| \leqq(4 r / \rho)^{2}
$$

and inserting (6) we arrive at (8).

To complete the proof we must construct specific surfaces for which equality is attained in (8). These turn out to be a slightly generalized form of the classical minimal surface of Enneper.

The construction which we use involves a representation formula having the property that the variable $\zeta$ of Lemma 1 associated with the surface represented is precisely the parameter used in the representation. We formulate this as a separate lemma.

LEMMA 3. Let $w(\zeta)$ be an arbitrary analytic function in a simply-connected region $D$ of the complex $\zeta$-plane. Assume that $D$ contains the origin. Define a surface $S$ parametrically by the equations

$$
\begin{aligned}
& x=\operatorname{Re} \int_{0}^{\zeta} \frac{1}{2}\left(1-w^{2}\right) d \zeta, \\
& y=\operatorname{Re} \int_{0}^{\zeta} \frac{i}{2}\left(1+w^{2}\right) d \zeta, \\
& z=\operatorname{Re} \int_{0}^{\zeta} w d \zeta .
\end{aligned}
$$

Then (a) $S$ is a minimal surface.

(b) To each point $\zeta$ in $D$ we have a corresponding point $q(\zeta)$ in $S$ and a complex number $w(\zeta)$. These are related as follows. If the normal to $S$ at $q(\zeta)$

(2) See note at the end of this paper. 
determines a point $\sigma(\zeta)$ on the unit sphere $\Sigma$ and if we project $\Sigma$ from the point $(0,0,1)$ onto the $x, y$-plane, then the image of the point $\sigma(\zeta)$ will have coordinates $\operatorname{Re} w(\zeta), \operatorname{Im} w(\zeta)$.

(c) If the element of arc length on $S$ is $d s$, then at each point $\zeta$ of $D$ we have $[d s /|d \zeta|]_{\zeta}=\left(1+|w(\zeta)|^{2}\right) / 2$.

REMARK. It may happen that distinct values $\zeta_{1}, \zeta_{2}$ give rise to the same point in space. Insofar as neighborhoods of $\zeta_{1}, \zeta_{2}$ map onto distinct neighborhoods in space we have the case of a simple self-intersection mentioned earlier, and we consider the images of $\zeta_{1}, \zeta_{2}$ to correspond to distinct points $q_{1}, q_{2}$ of $S$. So long as we do not have neighborhoods of $\zeta_{1}$ and $\zeta_{2}$ mapping onto the same neighborhood in space we can assign to each point $q$ of $S$ a unique point $\zeta$ in $D$ and a unique value $w(\zeta)$. This will certainly be the case if the map $w(\zeta)$ is one-to-one, since by property (b) above, distinct values of $\zeta$ will correspond to points with distinct normals.

Proof. That $S$ is a minimal surface follows from the fact that equations (10) are simultaneously a special case of the Weierstrass representation (see, for example, Rado [12, p. 28]) and of the representation given by $\operatorname{Bers}\left({ }^{3}\right)[1]$.

The verification of (b) and (c) is a simple computation, using the formula (10). If we denote by $X$ the vector $(x, y, z)$, and let $\zeta=\xi+i \eta, w=u+i v$, then we have the following formulas for the partial derivatives with respect to $\xi$ and $\eta$ :

$$
\begin{aligned}
& x_{\xi}-i x_{\eta}=\frac{d}{d \zeta} \int_{0}^{\zeta} \frac{1}{2}\left(1-w^{2}\right) d \zeta=\frac{1}{2}\left[1-u^{2}+v^{2}-2 i u v\right] \\
& y_{\xi}-i y_{\eta}=\frac{1}{2}\left[-2 u v+i\left(1+u^{2}-v^{2}\right)\right] \\
& z_{\xi}-i z_{\eta}=u+i v
\end{aligned}
$$

whence

$$
\begin{aligned}
& X_{\xi}=\frac{1}{2}\left(1-u^{2}+v^{2},-2 u v, 2 u\right), \\
& X_{\eta}=\frac{1}{2}\left(2 u v,-1-u^{2}+v^{2},-2 v\right) .
\end{aligned}
$$

We thus find

$$
\begin{aligned}
X_{\xi} \times X_{\eta} & =\frac{1+|w|^{2}}{2}\left(u, v, \frac{|w|^{2}-1}{2}\right), \\
\left|X_{\xi} \times X_{\eta}\right| & =\left(\frac{1+|w|^{2}}{2}\right)^{2} .
\end{aligned}
$$

(3) It was the geometric interpretation of this latter representation, pointed out to the author by P. Berg, which led to the present lemma. 
For the unit normal $N$ we have

$$
N=\frac{X_{\xi} \times X_{\eta}}{\left|X_{\xi} \times X_{\eta}\right|}=\left(\frac{2 u}{1+|w|^{2}}, \frac{2 v}{1+|w|^{2}}, \frac{|w|^{2}-1}{|w|^{2}+1}\right)
$$

and (b) follows from the standard formula (4) for stereographic projection. But

$$
\frac{d s^{2}}{|d \zeta|^{2}}=\left|X_{\xi} \times X_{\eta}\right|=\left(\frac{1+|w|^{2}}{2}\right)^{2}
$$

which proves (c).

Thus Lemma 3 is established and we may now use it to complete the proof of Theorem 1.

Given arbitrary real numbers $\beta, d$, with $0<\beta<\pi, d>0$, we wish to find a minimal surface for which equality is attained in (8). To do this we set $r=\tan (\beta / 2), \rho=6 d /\left(3+r^{2}\right)$, and let $S$ be the surface defined by equations (10) where $\zeta$ ranges over the whole plane, and $w=r \zeta / \rho$. For the point $p$ we choose the image of $\zeta=0$ and for $N$, the image of the disk $\Delta:|\zeta|<\rho$. We must show that the distance from $p$ to the boundary of $N$ is at least $d$. But any path $C$ from $p$ to the boundary of $N$ corresponds to a path $\Gamma$ from $\zeta=0$ to $|\zeta|=\rho$. Hence, if we set $|\zeta|=t$,

$$
\begin{aligned}
\int_{C} d s & =\frac{1}{2} \int_{\Gamma}\left(1+|w|^{2}\right)|d \zeta|=\frac{1}{2} \int_{\Gamma}\left(1+\frac{r^{2}|\zeta|^{2}}{\rho^{2}}\right)|d \zeta| \\
& \geqq \frac{1}{2} \int_{0}^{\rho}\left(1+\frac{r^{2} t^{2}}{\rho^{2}}\right) d t=\frac{\rho}{6}\left(3+r^{2}\right)=d .
\end{aligned}
$$

Here we have used property (c) of any surface given by (10). If we use property (b), and observe that the disk $\Delta:|\zeta|<\rho$ maps onto $N$ and onto $|w|<r$ $=\tan (\beta / 2)$, we see that all the normals to $N$ make an angle of less than $\beta$ with the direction of the negative $z$-axis. But the normal at $p$ points precisely in the direction of the negative $z$-axis since $p$ corresponds to the point $w=0$.

The hypotheses of Theorem 1 are therefore fulfilled. To compute the Gauss curvature $K$ at $p$ we use formula (3):

$$
|K|^{1 / 2}=\left[\frac{d \sigma}{d s}\right]_{p}=\frac{[d \sigma /|d w|]_{w=0} \cdot[|d w / d \zeta|]_{\zeta=0}}{[d s /|d \zeta|]_{\zeta=0}}=4 \frac{r}{\rho}=\frac{2 r\left(3+r^{2}\right)}{3 d} .
$$

This completes the proof of Theorem 1.

It may be worth noting that the surfaces for which we have shown that equality is attained in (8) have the property that the normals to points of $N$ fill out in one-to-one fashion the interior of a circle on the unit sphere $\Sigma$, with center at the point corresponding to the normal direction at $p$.

5. The general case. Throughout this section we shall use the following notation. By $N$ we shall again denote a simply-connected neighborhood of a 
point $p$ on a minimal surface. Let the distance along the surface from $p$ to the boundary of $N$ be at least $d$. The restriction which we make on $N$ in this section is that for some $\alpha>0$, all normals to points of $N$ make an angle of more than $\alpha$ with some fixed direction in space. Denote by $\omega$ the angle between the normal at $p$ and the fixed direction.

We shall assume that coordinates are chosen so that the fixed direction in space corresponds to the direction of the positive $z$-axis. If we make the Gauss normal map of $N$ in to the unit sphere $\Sigma$, then project stereographically into the $w$-plane, the image of $N$ will be included in the disk $|w|<r$, with $r=\cot (\alpha / 2)$, while the point $p$ will map onto a point $w_{0}$ with $a=\left|w_{0}\right|$ $=\cot (\omega / 2)$.

Finally, using Lemma 1 we find a map of $N$ into the $\zeta$-plane satisfying (2). We may assume that the point $p$ maps onto $\zeta=0$. Then we can again define the inverse map in a disk $|\zeta|<\rho$, and we obtain the following estimate.

LEMma 4. In the above notation we have

$$
\rho \geqq d / h(a, r)
$$

where

$$
\begin{aligned}
h(a, r)=\frac{1}{2 a^{3}}\left[2 r^{4} a\right. & -r^{3} a^{2}+\left(1-r^{2}\right) a^{3} \\
& \left.+r a^{4}+2 r^{3}\left(a^{2}-r^{2}\right) \log \left(1+\frac{a}{r}\right)\right], \quad \text { for } a>0,
\end{aligned}
$$

and

$$
\begin{aligned}
h(a, r)=\frac{1}{6}\left(3+r^{2}\right)+\frac{1}{4} r a+2 r^{2} \sum_{k=2}^{\infty} \frac{(-1)^{k}}{(k+1)(k+3)}\left(\frac{a}{r}\right)^{k}, & \\
0 & \leqq a<r .
\end{aligned}
$$

Proof. We note first of all that if $a=0$, this means that the normal at $p$ points in the direction of the negative $z$-axis, and the neighborhood $N$ satisfies the conditions of Lemma 2 with $\beta=\pi-\alpha$, and the same values of $d$ and $r$. But inserting $a=0$ in formula (13) we see that inequality (11) reduces to (6). (Note that formula (12) becomes indeterminate for $a=0$.)

We need therefore only consider the case where $a \neq 0$. The initial reasoning in Lemma 2 holds without change, since we again have an analytic map of $|\zeta|<\rho$ into $|w|<r$, and we obtain as before, inequality (7):

$$
d \leqq \int_{L} \frac{1+|w|^{2}}{2}|d \zeta|
$$

where $L$ is a radius of the disk $|\zeta|<\rho$. We must now obtain an estimate on $|w|$. We cannot apply Schwarz' Lemma directly since $\zeta=0$ now maps onto 
$w_{0} \neq 0$, but we may make a preliminary linear fractional transformation of $|w|<r$ which takes $w_{0}$ into the origin, apply Schwarz' Lemma, and transform back. The result is that for each value of $\zeta$ with $|\zeta|<\rho$, we have

$$
|w| \leqq \frac{r(r x+a)}{a x+r}, \text { where } x=\frac{|\zeta|}{\rho} .
$$

This yields

$$
\begin{aligned}
d & \leqq \int_{L} \frac{1+|w|^{2}}{2}|d \zeta| \leqq \frac{\rho}{2} \int_{0}^{1} \frac{\left(a^{2}+r^{4}\right) x^{2}+2 a r\left(1+r^{2}\right) x+r^{2}\left(1+a^{2}\right)}{a^{2} x^{2}+2 a r x+r^{2}} d x \\
& =\frac{\rho}{2 a^{3}}\left[\left(a^{3}+a r^{4}\right) x+2 r^{3}\left(a^{2}-r^{2}\right) \log (a x+r)-r^{2}\left(a^{2}-r^{2}\right)^{2} /(a x+r)\right]_{0}^{1} \\
& =\rho h(a, r)
\end{aligned}
$$

where $h(a, r)$ is given by (12).

Since the expression (13) is obtained merely by expanding the term $\log (1+(a / r))$ of $(12)$ in a power series, the lemma is proved.

THEOREM 2. Let $p$ be a point of a minimal surface and $N$ a simply-connected $\left({ }^{2}\right)$ neighborhood of $p$. Let the distance along the surface from $p$ to the boundary of $N$ be at least $d$. A ssume that for some $\alpha>0$ all normals to points of $N$ make an angle of at least $\alpha$ with some fixed direction, and let the angle between this fixed direction and the normal at $p$ be $\omega$. Set $a=\cot (\omega / 2), r=\cot (\alpha / 2)$. Then the Gauss curvature $K$ at $p$ satisfies

$$
|K| \leqq \frac{1}{d^{2}}\left[\frac{4\left(r^{2}-a^{2}\right) h(a, r)}{r\left(1+a^{2}\right)^{2}}\right]^{2}
$$

where $h(a, r)$ is again given by (12), (13).

Proof. By the Schwarz-Pick Lemma applied to the map of $|\zeta|<\rho$ into $|w|<r$, we have

$$
\left|\frac{d w}{d \zeta}\right|_{\zeta=0} \leqq \frac{r}{\rho}\left(1-\frac{|w(0)|^{2}}{r^{2}}\right)=\frac{1}{\rho} \frac{r^{2}-a^{2}}{r} .
$$

Applying (1) at the point $p$ gives

$$
|K|^{1 / 2}=\frac{4}{\left(1+a^{2}\right)^{2}}\left|\frac{d w}{d \zeta}\right|_{\zeta=0} \leqq \frac{1}{\rho} \frac{4\left(r^{2}-a^{2}\right)}{r\left(1+a^{2}\right)^{2}} .
$$

Inserting (11) we obtain (14), and the theorem is proved.

6. Special cases. We wish to examine now the form which inequality (14) takes in various special cases.

First of all we recall that the case $a=0$ corresponds precisely to the case of Theorem 1 where we set $\beta=\pi-\alpha$. But setting $a=0$ in equation (13) we find 


$$
h(0, r)=\frac{1}{6}\left(3+r^{2}\right)
$$

and inserting this in (14) we arrive at (8). Thus the inequality of Theorem 1 is a special case of Theorem 2 .

Next we note that the inequality (14) is a strict improvement over one obtained previously [10] under the same hypotheses as in Theorem 2. Namely, the function $h(a, r)$ was obtained by integrating with respect to a parameter a positive, strictly monotone increasing function of $a$. Using (12) we see that

$$
h(r, r)=\frac{1}{2}\left(1+r^{2}\right) .
$$

Hence for $a<r$ we have $h(a, r)<\left(1+r^{2}\right) / 2$, and inserting this inequality in (14) gives the result of [10].

Finally, we consider the case of a minimal surface of the form $z=f(x, y)$. We shall derive from (14) a very simple inequality for $K$ (inequality (25) below) which is weaker than (14) but which is still, as we shall show, not very far from being best possible.

For a neighborhood $N$ which is of the form $z=f(x, y)$ the normal directions omit at least half of the sphere. We therefore have $\alpha=\pi / 2, r=1$. We note that by our convention we are assuming that all normals are bounded away from the positive $z$-axis by more than $\pi / 2$ so that in this case we are choosing the normals to be directed in the negative $z$-direction. Thus the angle between the positive $z$-axis and that normal at $p$ which is directed upwards will be $\pi-\omega$, and if we denote by $W_{0}$ the value of $W=\left(1+f_{x}^{2}+f_{v}^{2}\right)^{1 / 2}$ at the point $p$, we have

$$
W_{0}=\sec (\pi-\omega)=\frac{1+a^{2}}{1-a^{2}} .
$$

Inserting this in (14) gives

$$
|K| \leqq \frac{1}{d^{2}} \frac{1}{W_{0}^{2}}[H(a)]^{2}
$$

where we have set

$$
H(a)=4 h(a, 1) /\left(1+a^{2}\right) .
$$

We show next that

$$
[H(a)]^{2}<8, \quad \text { for } 0 \leqq a<1 .
$$

We obtain this bound by dividing the interval $0<x<1$ in half, and bounding $h(x, 1)$ by a linear function in each half. 
We note first that by (13) the series expansion of $h(x, 1)$ is

$$
h(x, 1)=\frac{2}{3}+\frac{x}{4}+2 \sum_{k=2}^{\infty} \frac{(-1)^{k}}{(k+1)(k+3)} x^{k}, \quad 0 \leqq x<1 .
$$

Hence, for the derivative we have

$$
h^{\prime}(x, 1)=\frac{1}{4}+2 \sum_{k=2}^{\infty}(-1)^{k} \frac{k}{(k+1)(k+3)} x^{k-1}, \quad 0 \leqq x<1 .
$$

The power series on the right is an alternating series whose terms are decreasing in size, and since the first term is non-negative, we have $h^{\prime}(x, 1)$ $\geqq 1 / 4$ for $0 \leqq x<1$. Furthermore, by $(16), h(1,1)=1$. Hence $h(x, 1) \leqq(x+3) / 4$ for $0 \leqq x<1$. Thus

$$
H(x) \leqq F(x)=(x+3) /\left(x^{2}+1\right), \quad \text { for } 0 \leqq x<1 .
$$

But $F^{\prime}(x)<0$ for $x \geqq 1 / 2$, so that $F(x) \leqq F(1 / 2)=14 / 5$ for $1 / 2 \leqq x<1$. Hence

$$
[H(x)]^{2} \leqq(14 / 5)^{2}<8, \quad \text { for } 1 / 2 \leqq x<1 .
$$

We consider next the interval $0 \leqq x<1 / 2$. Using (21) we find

$$
h^{\prime \prime}(x, 1)=2 \sum_{k=2}(-1)^{k} \frac{k(k-1)}{(k+1)(k+3)} x^{k-2} .
$$

A calculation shows that the terms of this series decrease in size for $0 \leqq x<1 / 2$. Since the first term is positive we have $h^{\prime \prime}(x, 1)>0$ for $0 \leqq x<1 / 2$. But $h(0,1)$ $=2 / 3$ and using (12) we find that $h(1 / 2,1)<5 / 6$. Since the line passing through the points $(0,2 / 3)$ and $(1 / 2,5 / 6)$ has the equation $(x+2) / 3$, we have that $h(x, 1) \leqq(x+2) / 3$ for $0 \leqq x \leqq 1 / 2$, and hence

$$
H(x) \leqq G(x)=\frac{4}{3} \frac{x+2}{x^{2}+1}, \quad \text { for } 0 \leqq x \leqq 1 / 2 .
$$

But the maximum of $G(x)$ occurs at $x=5^{1 / 2}-2$; hence

$$
[H(x)]^{2} \leqq\left[G\left(5^{1 / 2}-2\right)\right]^{2}=4+16 \cdot 5^{1 / 2} / 9<8, \quad \text { for } 0 \leqq x \leqq 1 / 2 .
$$

Combining (24) with (22) we obtain inequality (19).

If we now insert (19) in (17) we arrive at the bound

$$
|K| \leqq \frac{8}{d^{2} W_{0}^{2}} .
$$

To test the accuracy of this inequality we note first of all that since $W_{0} \geqq 1$ in every case, inequality (25) implies

$$
|K| \leqq \frac{8}{d^{2}} \text {. }
$$


But when $W_{0}=1$ we have $a=0$ which corresponds to the case of Theorem 1 , and we know that for each value of $d$ there is a surface for which equality is attained in (8). Since in the present case we have $r=1$, this means that we can find a point $p$ and a neighborhood $N$ for which

$$
|K|=\frac{64}{9} \frac{1}{d^{2}} \text {. }
$$

Comparing this with (26) we see that the constant 8 in inequalities (25) and (26) cannot be much improved.

We should note that in Theorem 1 we have not excluded surfaces with self-intersections, so that the particular surface for which (27) holds might conceivably not be representable in the form $z=f(x, y)$. However, if one examines the surfaces obtained by setting $r=1$ in the proof of Theorem 1 , one can verify directly from equations (10) that the mapping of the $\zeta$-plane into the $x, y$-plane is one-to-one, so that the surface is indeed of the desired form.

As a final specialization of our inequality, we consider the case where the neighborhood $N$ lies over the disk $x^{2}+y^{2}<R^{2}$, with the point $p$ projecting onto the origin. We then have obviously $d \geqq R$ and it follows from (25) that the inequality

$$
|K| \leqq \frac{c}{R^{2} W_{0}^{2}}
$$

holds with $c=8$.

This inequality was originally proved by E. Hopf [4] with the constant $c$ equal to $8 / \mu$, where $\mu$ is Heinz constant [3]. The exact value of $\mu$ is not known, but the best estimate at present (see Nitsche [6], footnote on p. 270) is $\mu \geqq .64$ which would yield (28) with the constant $c=12.5$. Nitsche [7] gave a different proof of (28) with $c=12.25$. Both of these contain the original estimate of Heinz [3].

7. Other applications. Lemmas 2, 3, and 4 may be applied to many other problems besides the particular one treated in the previous sections. As one example, one can use Lemmas 2 and 4 to improve the results given in [11] for analytic functions on minimal surfaces, and using Lemma 3, one can show that certain of these results are best possible.

Lemma 3 turns out to be useful in answering many questions about the existence of minimal surfaces with preassigned properties. In particular, we note that the total curvature, or curvatura integra, is given by the total area of the image of the surface under the normal mapping into the unit sphere $\Sigma$.

Several comments on the total curvature of minimal surfaces were made in a previous paper [8]. The study of total curvature is relevant to the question of Bernstein's Theorem and possible generalizations, since it follows from a theorem of Blanc and Fiala [2] that a complete simply-connected 
minimal surface with finite total curvature must be conformally equivalent to the plane.

In this connection we note the following:

1. There exist complete simply-connected minimal surfaces (other than planes) with finite and with infinite total curvature.

2. There exists a simply-connected minimal surface of the form $z=f(x, y)$ with infinite total curvature.

Both of these statements follow immediately from Lemma 3. Namely, if we set $w=\zeta$ in (10) and let $\zeta$ vary over the whole plane, we obtain a complete simply-connected minimal surface whose total curvature is $-4 \pi$ since the normal map is a one-to-one map onto the sphere minus one point. On the other hand, if we set $w=e^{\zeta}$ in (10) and let $\zeta$ vary over the whole plane we obtain a surface with infinite total curvature, since the normal map will cover the entire sphere $\Sigma$ except for two points an infinite number of times. We may note that for an arbitrary choice of the function $w(\zeta)$ we will obtain a complete surface if we choose for the domain of $\zeta$ the whole plane, since condition (c) in Lemma 3 tells us that any curve on the surface will be at least half as long as its pre-image in the $\zeta$-plane, so that a curve going to the boundary will always have infinite length. An examination of this particular surface shows that points having the same normal direction, corresponding to values of $\zeta$ differing by an integer multiple of $2 \pi i$, will have different $y$-coordinates and hence be distinct points in space. Thus the surface can only have simple self-intersections, and it will indeed be a simply-connected complete surface with infinite total curvature.

The second statement above follows by choosing this same surface, but considering only that part of it which arises from values of $\zeta$ with $\operatorname{Re} \zeta<0$. In this case there will be no self-intersections and it can in fact be shown that the map from the $\zeta$-plane into the $x, y$-plane is one-to-one. Thus the surface may be written in the form $z=f(x, y)$, while the normal map into the sphere will cover every point of the lower hemisphere but one an infinite number of times.

In conclusion let us note that in the previous sections we have used only the most elementary properties of the map $w(\zeta)$. In fact all we have used has been Schwarz' Lemma in various forms. It is clear that by applying other results from function theory we can derive further properties of minimal surfaces. As our final example we follow a suggestion of M. Schiffer and obtain a lower bound on the Gauss curvature using the Koebe distortion theorem.

Let $p$ be a point of a minimal surface and let $N$ be a neighborhood of $p$ satisfying the conditions of Lemma 2. Suppose further that no two normals to $N$ point in the same direction. Then with $d, \beta$, and $r$, defined as in Lemma 2, set

$$
h=3 d /\left(3+r^{2}\right)
$$

and let $q$ be any point of $N$ whose distance to $p$ is $s \leqq h$. Let $K$ and $K_{0}$ be the 
Gauss curvatures at $q$ and $p$ respectively. Then

$$
|K|^{1 / 2} \geqq\left|K_{0}\right|^{1 / 2} \frac{1}{\left(1+r^{2} t^{2}\right)^{2}} \frac{1-t}{(1+t)^{3}}, \quad 0 \leqq t=\frac{s}{h}<1 .
$$

We omit the details of the proof, but note merely that the condition $s \leqq h$ is precisely what is needed, in view of (2) and (6), to guarantee that the point $q$ maps onto a point $\zeta_{1}$ with $\left|\zeta_{1}\right|<\rho$. Since the map of $|\zeta|<\rho$ into $|w|<r$ is now one-to-one, we obtain by Koebe's distortion theorem a lower bound for $|d w / d \zeta|$ at $\zeta_{1}$ in terms of $|d w / d \zeta|$ at $\zeta=0$, and using (1) we arrive at (30).

As a final remark, we note that inequality (30) is trivial if $K_{0}=0$. On the other hand, if $K_{0} \neq 0$, then the map of the surface into the w-plane is one-to-one in a neighborhood of $p$, so that the hypotheses are satisfied for a suitable choice of $N$. Thus given an arbitrary point of a minimal surface there always exists a neighborhood in which (30) is satisfied. Clearly, the larger we can make $d$, keeping $\beta$ small, the better inequality (30) becomes.

Added in proof (May 6, 1960). The main results of this paper, and in particular Theorems 1 and 2, remain valid without assuming that the neighborhood $N$ is simply connected. One need only apply in a suitable manner the reasoning given here to the universal covering surface of $N$. This, and related questions, will be discussed in a paper on the global theory of minimal surfaces, to appear in the Commentarii Mathematici Helvitici.

\section{BIBLIOGRAPHY}

1. L. Bers, Isolated singularities of minimal surfaces, Ann. of Math. vol. 53 (1951) pp. 364386.

2. C. Blanc and F. Fiala, Le type d'une surface et sa courbure totale, Comment. Math. Helv. vol. 14 (1941-1942) pp. 230-233.

3. E. Heinz, Über die Lösungen der Minimalflächengleichung, Nachr. Akad. Wiss. Göttingen Math.-Phys. Kl. (1952) pp. 51-56.

4. E. Hopf, On an inequality for minimal surfaces $z=z(x, y)$, J. Rat. Mech. Anal. vol. 2 (1953) pp. 519-522.

5. J. Nitsche, Über eine mit der Minimalflüchengleichung zusammenhängender analytische Funktion und der Bernstein'schen Satz, Arch. Math. vol. 7 (1956) pp. 417-419.

6. - On harmonic mappings, Proc. Amer. Math. Soc. vol. 9 (1958) pp. 268-271.

7. On an estimate for the curvature of minimal surfaces $z=z(x, y), \mathrm{J}$. Math. Mech. vol. 7 (1958) pp. 767-770.

8. R. Osserman, Remarks on minimal surfaces, Comm. Pure Appl. Math. vol. 12 (1959) pp. 233-239.

9. - Proof of a conjecture of Nirenberg, Comm. Pure Appl. Math. vol. 12 (1959) pp. 229-232.

10. - An analogue of the Heinz-Hopf inequality, J. Math. Mech. vol. 8 (1959) pp. 383-385.

11. - An extension of certain results in function theory to a class of surfaces, Proc. Nat. Acad. Sci. U.S.A. vol. 45 (1959) pp. 1031-1035.

12. T. Radó, The problem of Plateau, Ergebn. d. Math. vol. 2(2), Berlin, 1933.

STANFORD UNIVERSITY, Stanford, California 